\title{
Redox-Sensitive Cross-Linking Enhances Albumin Nanoparticle Function as Delivery System for Photodynamic Cancer Therapy
}

Anna M Molina1 ${ }^{1}$ Moraima Morales-Cruz², Marimar Benítez², Kiara Berríos ${ }^{1}$, Cindy M Figueroa ${ }^{1}$ and Kai Griebenow ${ }^{2 *}$

${ }^{1}$ Department of Chemistry, University of Puerto Rico, Río Piedras Campus, San Juan, PR 00931, USA

${ }^{2}$ Department of Biology, University of Puerto Rico, Río Piedras Campus, San Juan, PR 00931, USA

\begin{abstract}
Photodynamic cancer therapy is still limited in its efficiency because of a lack of targeted methods avoiding nonspecific toxicity. To overcome this we developed a system that is solely effective upon cellular uptake and intracellular activation by incorporating redox-sensitive chemistry. We used a nanoprecipitation method to obtain human serum albumin nanoparticles (HSA NP) with a diameter of $295 \pm 5 \mathrm{~nm}$ and decorated them with the photosensitizer (PS) chlorin e6 (Ce6). The NP was stabilized using a redox-sensitive cross-linker to create a smart drug delivery system that is activated only upon NP disintegration in the reducing intracellular environment. Indeed, our drug delivery NP broke down in an environment emulating the reducing intracellular environment with $10 \mathrm{mM}$ glutathione, but not under extracellular conditions. In contrast, the control cross-linked with glutaraldehyde did not break down in the reducing environment. Upon NP disintegration $\mathrm{Ce} 6$ fluorescence doubled as the result of diminished self-quenching. While the Ce6-HSA NP did not produce a significant amount of singlet oxygen upon irradiation, NP disintegration restored singlet oxygen production to about half of the value generated by the free Ce6. In vitro experiments with HeLa cells showed that the smart system was able to kill up to $81 \%$ of the cells while the glutaraldehyde cross-linked control only killed $56 \%$ of them at a drug concentration of $10 \mathrm{ng} / \mathrm{ml}$. Also, Ce6 immobilization in HSA NP prevented dark toxicity in two different cell lines. For the first time, we demonstrate that it is possible to design a smart NP drug delivery system delivering a PS drug to cancer cells while avoiding toxicity prior to the uptake and irradiation. This finding may provide a means of designing more efficient PDT in cancer treatment.
\end{abstract}

Keywords: Albumin nanoparticles; Chlorin e6; Drug delivery system; Photodynamic therapy; Redox-responsive

\section{Introduction}

Cancer is a heterogeneous group of diseases traditionally treated by chemo and radiotherapy, with different success rates for different types of cancer. However, there are many drawbacks to overcome in traditional therapies, such as generalized drug toxicity and drug resistance $[1,2]$. Photodynamic therapy (PDT) is a promising technology in this context $[3,4]$. It relies in the accumulation of photosensitizer drugs in a target area and the subsequent application of irradiation in the presence of oxygen. Upon irradiation, the photosensitizer generates reactive oxygen species (ROS), causing oxidative damage and ultimately, cell death. The resulting damage is localized, given the reactive nature of the ROS [5].

Photosensitizers have been successfully optimized to attain higher efficiencies at longer wavelengths to enhance light penetration in tissue, one of the greatest challenges of PDT [6]. Currently, research focuses on the conjugation of photosensitizers to delivery systems that increase selectivity for tumor tissues over healthy tissues and decrease dark toxicity [7-10]. The drawbacks of most photosensitizers include inappropriate tissue retention and activation energies, dark toxicity, hydrophobicity, and lack of specificity, most of which can be improved with an efficient delivery system [4]. Nanoparticles (NP) are being explored in this work as delivery systems for a model photosensitizer drug to target solid tumors.

It is generally agreed that particles within a certain size range can penetrate and accumulate in solid tumors because the tumor vasculature is leaky and tumor tissue often lacks efficient lymphatic drainage. The phenomenon of passive accumulation of NP in tumors is known as the enhanced permeability and retention (EPR) effect [11-13]. In general, NP from 100 to $800 \mathrm{~nm}$ can enter the irregular tumor fenestrae, but must be bigger than $20 \mathrm{~nm}$ to avoid renal filtration [14,15].
Human serum albumin (HSA) NP have been used as drug delivery systems [16-19]. HSA is the most abundant protein in human plasma and plays a determinant role in the transport of many metabolic compounds and drugs [20]. It is very robust and remains stable over a $\mathrm{pH}$ range of $4-9$ and at high temperatures $\left(60^{\circ} \mathrm{C}\right)$. Because it is easily available, biodegradable, has a long blood half-life, and does not produce an immune response, it is very attractive as a drug carrier. Moreover, it has been found to preferentially accumulate in tumor and inflamed tissue [21], a property being exploited in the FDA approved anti-cancer drug Abraxane ${ }^{\infty}$.

Recent studies have investigated the feasibility of HSA NP as delivery systems for PDT [22-24]. The systems described thus far exclusively rely on drug binding by adsorption. A significant drawback of this type of binding is that the leaching of the drug from the carrier can occur before effective accumulation in the target tissue takes place. A comparative study of PS-loaded and conjugated glycol chitosan NP found that covalently bound drug resulted in enhanced efficiency in vivo over physically loaded drug due to its prolonged circulation time and higher accumulation in the tumor site [25].

The model photosensitizer drug selected for this study was Chlorin e6 (Ce6), a second-generation photosensitizer activatable

*Corresponding author: Griebenow K, Department of Biology, University of Puerto Rico, Río Piedras Campus, San Juan, PR 00931, USA, Tel: 787-7640000/7374, 787-522-1392; Fax: 787-722-1390; E-mail: kai.griebenow@gmail.com

Received April 17, 2015; Accepted May 11, 2015; Published May 22, 2015

Citation: Molina AM, Morales-Cruz M, Benítez M, Berríos K, Figueroa CM, et al (2015) Redox-Sensitive Cross-Linking Enhances Albumin Nanoparticle Function as Delivery System for Photodynamic Cancer Therapy. J Nanomed Nanotechnol 6: 294. doi:10.4172/2157-7439.1000294

Copyright: (c) 2015 Molina AM, et al. This is an open-access article distributed under the terms of the Creative Commons Attribution License, which permits unrestricted use, distribution, and reproduction in any medium, provided the original author and source are credited. 
by near-infrared wavelengths [26]. This drug was selected due to its high photodynamic efficiency as well as the available moieties for covalent coupling to the drug delivery system. The coupling of the photosensitizer to a NP or another macromolecule generally results in non-photochemical excitation quenching which drastically reduces the generation of singlet oxygen. This quenching-induced deactivation of the photosensitizers can be reversed in vitro, for example by degradation of the delivery system into smaller units [27]. Herein we sought to exploit this and developed a drug delivery system involving stimulus-responsive (thiol-cleavable) cross-linking. The system was using the cross-linker to form stable bonds in solution under aerobic conditions preventing disintegration of the protein NP in the aqueous environment. However, we aimed at designing the system in a manner that exposure to a reducing environment, typical for the interior of cells, would lead for the NP to break down. This in turn should lead to reduced excitation quenching of the photosensitizer and improve generation of singlet oxygen upon irradiation. Because the cytosol of cells is much more reducing than the extracellular space, a thiolcleavable cross-linker is an attractive candidate for intracellular drug release/activation. In this study we systematically compare the Ce6HSA NP cross-linked with a thiol-cleavable cross-linker with particles prepared using glutaraldehyde, which leads to redox-insensitive crosslinking.

\section{Materials and Methods}

Chlorin e6 (Ce6) was purchased from Frontier Scientific, Logan, UT. HSA, ethanol $(\geq 99.5 \%)$, DMSO $(\geq 99.9 \%)$, glutaraldehyde (25\% in water), 1-ethyl-3-[3-dimethylaminopropyl]carbodiimide hydrochloride (EDC), N-hydroxysuccinimide (NHS), and reduced glutathione ethyl ester were from Sigma-Aldrich (St. Louis, MO). Dithiobis[succinimidyl propionate] (DSP) was from Thermo, Waltham, MA. 4', 6-Diamidino-2-phenylindole (DAPI), propidium iodide (PI), and FM-4-64 membrane stain were purchased from Invitrogen (Grand Island, NY). All reagents were used without further purification. HeLa and HUVEC cells were purchased from the American Type Culture Collection (Manassas, VA).

\section{Preparation of the HSA-Ce6-FA NP}

Synthesis of the NHS ester of Ce6: The Ce6 NHS-ester was synthesized by reacting 2.5 equivalents of EDC and 5 equivalents of NHS with 1 equivalent of Ce6 in a solution of 2:1 ethanol:MES buffer (vol:vol) at a $\mathrm{pH}$ of 6.0. The product of this reaction was precipitated by the addition of MES buffer followed by centrifugation for the removal of excess EDC and NHS.

Cross-linking of the HSA NP: The HSA NP were prepared by a modified method according to Langer [19]. Briefly, HSA was dissolved in $10 \mathrm{mM} \mathrm{NaCl}, \mathrm{pH} 9.0$, to achieve a final concentration of $25 \mathrm{mg} / \mathrm{ml}$. Ethanol was added at a constant rate of $1 \mathrm{ml} / \mathrm{min}$ with an automated syringe to reach a final ratio of 1-to-4 water-to-ethanol under stirring. Then, either dithiobis[succinimidyl propionate] (DSP) (Thermo, Waltham, MA) in DMSO or glutaraldehyde (25\%) (Sigma-Aldrich, St. Louis, MO) in water was added to achieve NP cross-linking. Since the NP consist of a water-soluble protein the cross-linking was essential to prevent dissolution of the NP upon exposure to an aqueous environment (e.g. reconstitution buffer, blood).

Surface modification of HSA NP with Ce6: The purified Ce6 NHS-ester was dissolved in pure ethanol and its concentration was determined from its absorbance at $400 \mathrm{~nm}$ using the extinction coefficient of $211.16 \mathrm{mg} / \mathrm{ml}$ and $1 \mathrm{~cm}$ path length. The reaction product was then added to the NP suspension at a molar ratio of 1-to-15 HSA-to-Ce6 to react overnight. The modified NP were centrifuged at $11000 \mathrm{rpm}$, resuspended in water, and the supernatant discarded. This washing step was repeated twice to remove drug that was not covalently attached. The drug loading was determined by measuring the UV/Vis absorption at $400 \mathrm{~nm}$ of the supernatants discarded and subtracting it from the initial Ce6 NHS-ester concentration.

\section{Physical characterization of the HSA-Ce6-FA NP}

Size, polydispersity, zeta potential, and morphology: The size, polydispersity, and zeta potential of the Ce6-HSA NP were determined by dynamic light scattering (DLS) using a Malvern Zetasizer Nanoseries. The samples were dispersed in nanopure water and sonicated until a good suspension was obtained. The morphology was examined by a JEOL $5800 \mathrm{LV}$ scanning electron microscopy (SEM) at $20 \mathrm{kV}$. The samples were coated with gold for $10 \mathrm{sec}$ in a Denton Vacuum DV502A.

Degree of NP cross-linking: The average number of amines of the HSA modified by DSP was determined by the 2,4,6-trinitrobenzene sulfonic acid (TNBSA) chromogenic assay after disintegration of the NP with a DTT solution [28].

NP disintegration and fluorescence: The disintegration of the NP was evaluated by a turbidity assay. Briefly, the glutaraldehydecross-linked Ce6-HSA NP and the DSP-cross-linked Ce6-HSA NP were incubated in a $10 \mathrm{mM}$ glutathione solution. Aliquots of each suspension were retrieved at predetermined times and the absorbance at $700 \mathrm{~nm}$ was measured. It has to be noted that the absorbance of Ce6 is negligible at $700 \mathrm{~nm}$ while significant at shorter wavelengths. The absorption was plot against time and normalized to $100 \%$ turbidity at $\mathrm{t}=0$. To evaluate the changes in fluorescence intensity, the NP were suspended in a reducing media and the fluorescence spectra were obtained at predetermined times using a Varian Cary Eclipse Fluorescence Spectrophotometer.

Singlet oxygen generation: The generation of singlet oxygen by DSP-cross-linked Ce6-HSA NP, glutaraldehyde-cross-linked Ce6HSA NP, and free Ce6 was determined by the p-nitrosodimethylaniline (RNO) bleaching method [29-31]. Singlet oxygen reacts with imidazole to form intermediate products that proportionally oxidize RNO, bleaching its absorbance at $440 \mathrm{~nm}$. Briefly, the Ce6-HSA NP crosslinked with either DSP or glutaraldehyde were suspended in phosphate buffer ( $\mathrm{pH}=7.4)$ in reducing (10 $\mathrm{mM}$ glutathione) or non-reducing conditions. The suspensions were mixed with imidazole $(10 \mu \mathrm{M})$ and RNO $(50 \mu \mathrm{M})$. Immediately afterwards, they were irradiated with a LED lamp $\left(\lambda_{\max }=660 \pm 10 \mathrm{~nm}\right)$ at a fixed distance of $5 \mathrm{~cm}$ and a fluence rate of $50 \mathrm{~mW} / \mathrm{cm}^{2}$, while bubbling with oxygen. Aliquots were retrieved every five minutes, centrifuged (11,000 rpm, 1 minute), and the supernatant absorbance was measured at $440 \mathrm{~nm}$. The results were normalized to abs $=0$ at $\mathrm{t}=0$.

\section{In vitro experiments}

Cell culture: HeLa cells (human epithelial cervix adenocarcinoma) and HUVEC cells (human endothelial umbilical vein normal cells) were cultured according to the instructions given by the American Type Culture Collection. The cells were grown in minimum essential medium (MEM) or Ham's F-12 medium containing 1\% L-glutamine, $10 \%$ fetal bovine serum (FBS), and $1 \%$ penicillin in a humidified incubator with $5 \% \mathrm{CO}_{2}$ and $95 \%$ air at $37^{\circ} \mathrm{C}$. All experiments were conducted before the cells reached 20 passages. 
Cell viability assay: HeLa or HUVEC cells were seeded in 96-well plates for $24 \mathrm{~h}$ in MEM or Ham F-12 containing 1\% L-glutamine, 10\% FBS, and $1 \%$ penicillin. The cell growth was then arrested by decreasing the FBS concentration in the medium to $1 \%$ for $18 \mathrm{~h}$. Subsequently, the cells were washed with PBS, and incubated with the glutaraldehyde or DSP-cross-linked Ce6-HSA NP at varying concentrations $(5-50 \mathrm{ng} / \mathrm{ml})$ and incubation times $(1,6$, or $24 \mathrm{~h})$. For photo toxicity experiments, the cells were irradiated with a LED lamp $\left(\lambda_{\max }=660 \pm 10 \mathrm{~nm}\right)$ placed at a distance of $8 \mathrm{~cm}$ from the cell plate immediately after incubation for a total light dose of $3 \mathrm{~J} / \mathrm{cm}^{2}$. For cell viability measurements, the CellTiter 96 aqueous nonradioactive cell proliferation assay (Promega, Madison, WI) was used. $20 \mu \mathrm{L}$ of 3-(4,5-dimethylthiazol-2-yl)-5-(3 carboxymethoxyphenyl)-2-(4-sulfophenyl)-2H-tetrazolium, inner salt (MTS) and phenazine methosulfate (PMS) was added to each well (333 $\mu \mathrm{g} / \mathrm{mL}$ MTS and $25 \mu \mathrm{M}$ PMS) and after $1 \mathrm{~h}$ the absorbance at $492 \mathrm{~nm}$ was measured using a microplate reader. Untreated cells were used as a negative control.

Confocal microscopy-apoptosis induction: HeLa cells were seeded as described before in lab-tek chambered coverslides (4 wells) for their examination by confocal laser scanning microscopy (CLSM). The cells were incubated with the glutaraldehyde or the DSP-crosslinked Ce6-HSA NP at a Ce 6 concentration of $10 \mathrm{ng} / \mathrm{ml}$ for $6 \mathrm{~h}$ and irradiated for $10 \mathrm{~min}$ exactly as described above for a total light dose of $1.5 \mathrm{~J} / \mathrm{cm}^{2}$. The cells were washed with PBS $(2 \mathrm{x}, 3 \mathrm{~min})$ and incubated with DAPI $(300 \mathrm{nM})$ and next with PI $(75 \mu \mathrm{M})$ for 5 min each. A solution of $3.7 \%$ formaldehyde was used to fix the cells. The HeLa cells were examined under a Zeiss laser scanning microscope 510 using a $67 \times$ objective. DAPI was excited at $405 \mathrm{~nm}$ and its emission was detected at $420-480 \mathrm{~nm}$. PI was excited at $561 \mathrm{~nm}$ and its emission was detected at $600-674 \mathrm{~nm}$.

Confocal microscopy - endosomal escape: The internalization of the bioconjugates and their ability to escape endosomal entrapment were determined by confocal laser scanning microscopy (CLSM). The glutaraldehyde and the DSP-cross-linked Ce6-HSA NP were labeled with FITC. The cells were incubated with FITC-labeled Ce6-HSA NP at a drug concentration of $10 \mathrm{ng} / \mathrm{ml}$ and an endosome marker (FM$4-64 ; 10 \mu \mathrm{g} / \mathrm{mL}$ ) at $37^{\circ} \mathrm{C}$ for $24 \mathrm{~h}$ in the dark. Then the medium was removed and the cells were washed with PBS three times, and fixed with $3.7 \%$ formaldehyde. The coverslips were examined as described before and excited at $488 \mathrm{~nm}$. FITC-MSN fluorescence was detected at wavelengths between 513 and $588 \mathrm{~nm}$ and the endosome marker between 598 and $738 \mathrm{~nm}$.

\section{Statistical analysis}

The relative cell viability (\%) was calculated by the formula: relative cell viability $(\%)=($ Abs test sample/Abs untreated cells $) \times 100$. The results were expressed as mean \pm SD. Mann Whitney analysis was used for comparison of two independent groups for cell viability in dark toxicity experiments using Stata ${ }^{\circledR}$ software. Difference between control (untreated cells) and experimental group (glutaraldehyde and DSP cross-linked NP) was considered statistically significant at $\mathrm{p}<0.05$. Two tailed t-test analysis was performed for comparison of two independent groups using SigmaPlot 12.0 software in time- and dose-dependent cell viability experiments. A p-value $<0.05$ was considered statistically significant within the $95 \%$ confidence interval. In all experiments, $\mathrm{n}=8$.

\section{Results}

PDT ideally has dual selectivity: first, it can preferentially be accumulated in the target tissue, and second, oxidative damage is induced only locally by irradiation. Enhanced tumor selectivity is usually accomplished by immobilization of the photosensitizers in a designed drug delivery system. The covalent immobilization of the photosensitizer in a suitable NP system is an example and this may also prevent or attenuate prolonged skin sensitivity and facilitate clearance $[32,33]$. In this paper, we utilized albumin NP to bind the photosensitizer. We hypothesized that during delivery, self-quenching should reduce cytotoxic effects by accidental radiation. The NP were designed using a redox-sensitive cross-linker, and we hypothesized that they should disintegrate and thus eliminating the self-quenching only after uptake by the target cells (Scheme 1).

Several methods have been published to obtain protein NP [1618]. However, even though the obtained size was desirable for our application (a diameter of $90-250 \mathrm{~nm}$ ), a broad size distribution was observed. Langer optimized a nanoprecipitation procedure to obtain suitable NP with a narrow size distribution [19]. Thus, the HSA NP were prepared by us using this enhanced nanoprecipitation method followed by cross-linking with either DSP or glutaraldehyde. DSP has a thiol group in the spacer arm which can be broken by reduction, while the glutaraldehyde forms a stable covalent bond that is not redoxsensitive. The NP thus obtained were modified with the purified Ce6 NHS-ester as described in the methods section at a molar ratio of 15to-1 Ce6-to-HSA during the overnight reaction. This resulted in the end in an about 9:1 molar ratio of Ce6-to-HSA (Table 1).

The Ce6-HSA NP synthesized by us were $<300 \mathrm{~nm}$ in diameter with a low polydispersity and a negative zeta potential (Table 1). No significant difference was observed in the nanoparticle size and the determined drug loading between the glutaraldehyde and the DSPcross-linked nanoparticles, but an increase in polydispersity and negative surface zeta potential was produced when cross-linking with DSP instead of glutaraldehyde prior to immobilization of Ce6. Optical inspection by SEM showed spherical particles with a narrow size distribution and a smooth surface (Figure 1). The general characteristics of the synthesized system are similar to other albuminbased NP previously reported [34].

A critical examination of the DSP-cross-linked Ce6-HSA NP consisted in testing whether they would disintegrate in a reducing environment and if this would indeed result in a decrease in the photo quenching. To test this, we synthesized three preparations of HSA NP varying the amount of cross-linking by using the DSP cross-linker at

\begin{tabular}{|l|l|l|l|l|}
\hline NP cross-linker & $\begin{array}{l}\text { Diameter } \\
(\mathbf{n m})\end{array}$ & $\begin{array}{l}\text { Polydispersity } \\
\text { Index }\end{array}$ & $\begin{array}{l}\text { Zeta potential } \\
(\mathbf{m V})\end{array}$ & $\begin{array}{l}\text { Ce6 loading (Ce6/ } \\
\text { HSA molar ratio) }\end{array}$ \\
\hline Glutaraldehyde & $298 \pm 6$ & $0.13 \pm 0.01$ & $-12.6 \pm 0.3$ & $9.6 \pm 0.9$ \\
\hline DSP & $\mathbf{2 9 5} \pm \mathbf{5}$ & $\mathbf{0 . 2 4} \pm \mathbf{0 . 0 2}$ & $\mathbf{- 1 8 . 0 \pm 0 . 6}$ & $\mathbf{9 . 1} \pm \mathbf{0 . 7}$ \\
\hline
\end{tabular}

Table 1: Characteristics of Ce6-HSA NP cross-linked with glutaraldehyde or DSP.
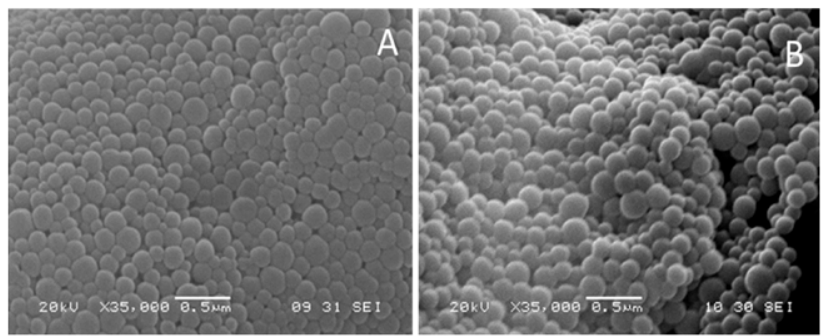

Figure 1: Scanning electron microscopy (SEM) images of NP cross-linked with glutaraldehyde (A) and DSP (B). 


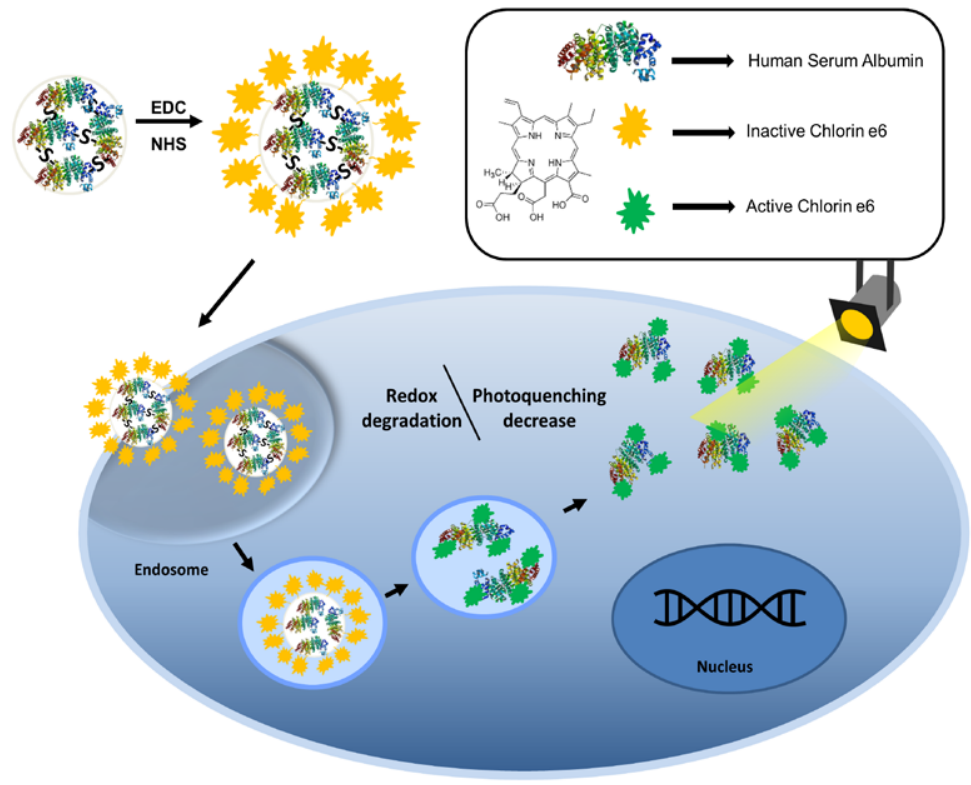

Scheme 1: Representation of the redox-sensitive cross-linked photosensitizer-protein nanoparticles and possible internalization and activation routes.
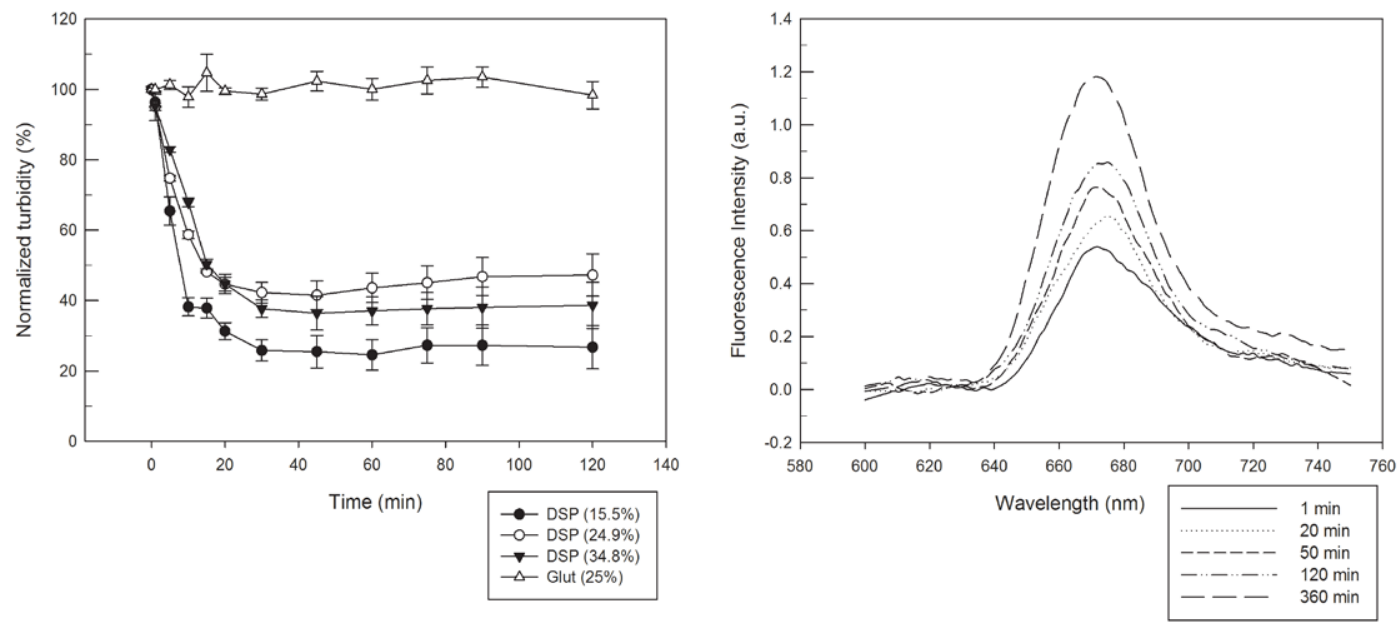

Figure 2: A: UV/Vis turbidity assay to determine the disintegration rate of HSA NP after exposure to $10 \mathrm{mM}$ glutathione. B: Changes in fluorescence of the DSP-cross-linked HSA NP exposed to $10 \mathrm{mM}$ glutathione. When DSP-cross-linked NP were exposed to redox conditions corresponding to extracellular conditions $(0.001 \mathrm{mM}$ glutathione), no significant changes in fluorescence intensity were detected.

different concentrations. To estimate the extent of cross-linking with DSP, the amount of albumin amines modified with the cross-linker was determined by using the TNBSA assay. Under our conditions, the amount of amines modified per albumin molecule were 16,24 , and $35 \%$, respectively, of the available amines. These DSP-cross-linked Ce6-HSA NP preparations were then examined for their ability to disintegrate in a reducing environment simulating the interior of cells. The Ce6-HSA NP were suspended in phosphate buffer containing $10 \mathrm{mM}$ glutathione which corresponds to its concentration inside of cells [35]. The turbidity of the suspensions was measured after various incubation times. The decrease in turbidity is proportional to the NP disintegration. All of the Ce6-HSA NP stabilized using the redoxresponsive cross-linker showed a marked decrease in turbidity in the glutathione solution, while the glutaraldehyde-cross-linked Ce6HSA NP showed no decrease in turbidity (Figure 2A). The amount of amines per albumin molecule modified with the redox-sensitive crosslinker increased proportionally to the amount of cross-linker added to the reaction. As expected, a higher modification degree resulted in a slightly increased stability in a reducing environment; hence, the nanoparticles took longer to break down. Because the NP with $15.5 \%$ of its amines cross-linked with DSP yielded the fastest disintegration while leaving a greater number of amines available for further reactions, this preparation was selected and used in all further studies.

Next we investigated whether the disintegration of the NP after exposure to reducing agents would produce the anticipated decrease of 
Ce6 photo quenching. Non-chemical quenching of the photosensitizer fluorescence results in shorter fluorescence lifetimes [36] and thus fluorescence yields. Thus, a reduction in quenching should cause an increase in the observed fluorescence emission under otherwise constant conditions. Indeed, we observed an increase in the Ce6 fluorescence emission after NP incubation in a solution containing 10 $\mathrm{mM}$ glutathione (Figure $2 \mathrm{~B}$ ). The two experiments assessing particle size and fluorescence intensity validated our general design ideas. While no changes were observed for the glutaraldehyde-cross-linked particles, particles cross-linked with the cleavable linker showed a decrease in particle size and an increase in fluorescence intensity upon establishing intracellular redox conditions.

Next, we tested whether the observed photo quenching decrease would effectively translate into an increased ROS production following irradiation. Upon excitation $(h v)$ of the photosensitizer intersystem crossing may occur resulting in the generation of ROS by two possible mechanisms. Type I reactions involve the formation of radical intermediates that transfer their energy to oxygen to form the superoxide anion radical $\left(\mathrm{O}_{2}^{-}\right)$and the hydroxyl radical $\left(\mathrm{OH}^{-}\right)$. Type II reactions consist in the transfer of energy from the photosensitizer excited state to the triplet ground state of molecular oxygen $\left({ }^{3} \mathrm{O}_{2}\right)$ to produce singlet oxygen $\left({ }^{1} \mathrm{O}_{2}\right)$. Because the lifetime of the triplet excited state $\left(\mathrm{T}_{1}\right)$ is longer than that of the singlet state $\left(\mathrm{S}_{1}\right)$, the type II mechanism typically predominates [7]. Even though tumor destruction by PDT is complex (including apoptosis and necrosis, as well as induction of immunological responses and destruction of the tumor vasculature), singlet oxygen is considered the main cytotoxic agent $[37,38]$.

To test for singlet oxygen production of the Ce6-HSA NP after exposure to reducing and non-reducing conditions, the p-nitrosodimethylaniline (RNO) bleaching method was used [29,30]. Both glutaraldehyde and DSP-cross-linked Ce6-HSA NP were used to bleach RNO in PBS in a non-reducing medium and after being exposed to a reducing medium for $18 \mathrm{~h}$. Unconjugated Ce6 was used as an additional control in this set of experiments. Our data show that the covalent attachment of $\mathrm{Ce} 6$ to the NP effectively prevented the generation of singlet oxygen because no significant bleaching of the dye occurred (Figure 3). In contrast, the control consisting in free Ce6 produced the highest decrease in absorbance of RNO as result of copious singlet oxygen generation. Importantly, when the smart NP were exposed to reducing agents and disintegrated, the singlet oxygen generation increased to approximately half of the value produced by the free Ce6. Glutaraldehyde-cross-linked NP did not show such a significant increase in singlet oxygen production when exposed to reducing conditions. This highlights a desirable characteristic of a smart drug delivery system for photosensitizers: prevention of premature toxicity even upon (accidental) premature irradiation. Our system clearly fulfills this requirement.

Even though a drug delivery system is expected to minimize nonselective cell internalization, the targeting of healthy cells is always a concern. To address this issue we tested the dark toxicity (cell viability without irradiation) of the photosensitizer-protein NP and the free drug in both HeLa cells (cervical cancer) and human umbilical vein endothelial cells (HUVEC), a normal (non-cancer) cell line. The results show that even at the lowest concentration tested the free drug caused some toxicity, which was significantly reduced by the drug immobilization in the HSA NP. The free drug dose toxic effect was more evident in the normal cell line tested (Figure 4). Neither the glutaraldehyde nor the DSP-cross-linked NP caused significant decrease in cell viability at any concentration tested by us. These results are further evidence of the advantage of placing the photosensitizer in a delivery system instead of administering the free drug. It is possible that the system selectivity towards specific cell types can be further enhanced by modifying the NP surface with a targeting ligand.

Next, we wanted to investigate the mechanism of cell death caused by NP-mediated PDT. For this purpose, HeLa cells were treated

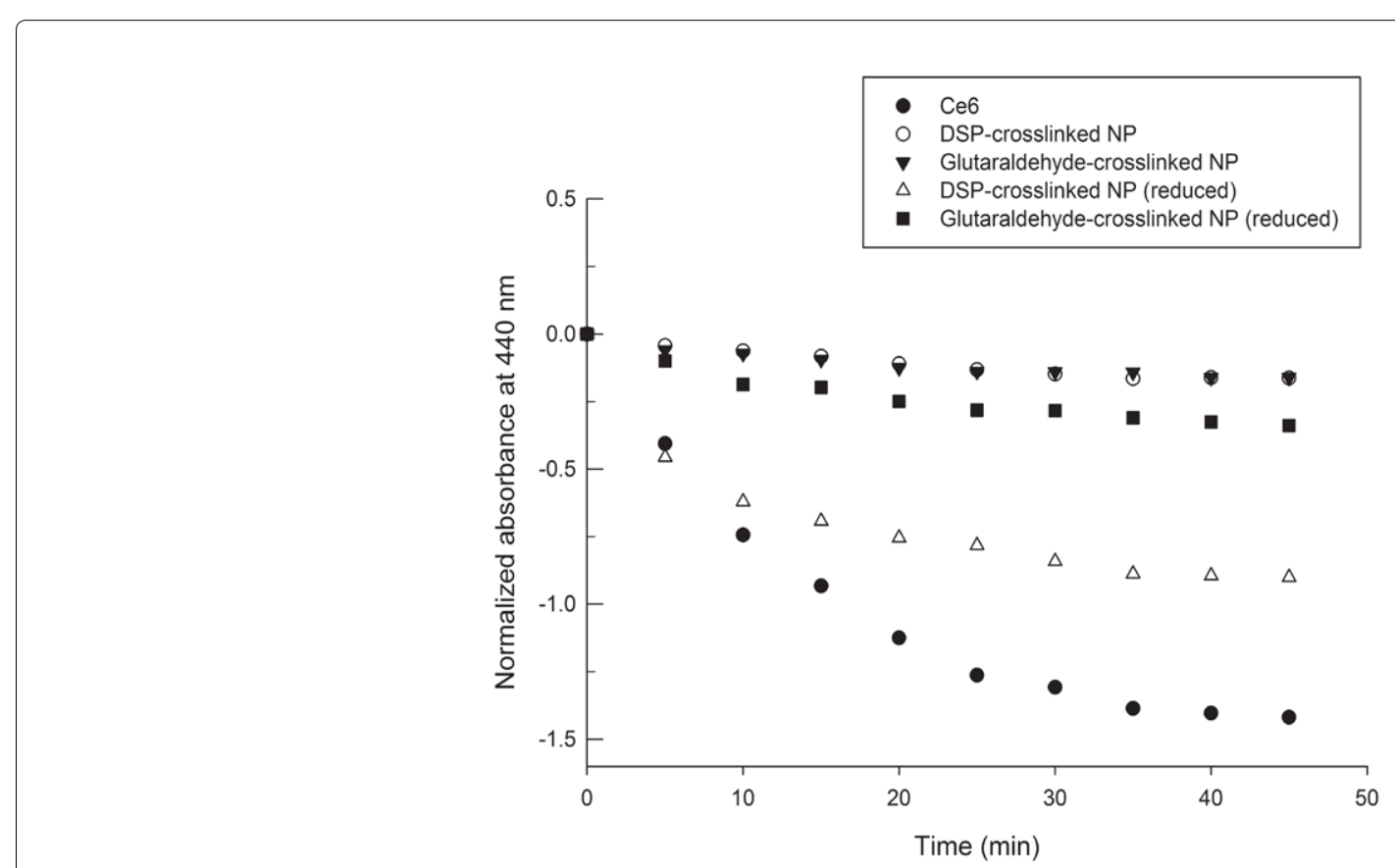

Figure 3: Spectrophotometric assay for the determination of singlet oxygen generation by the Ce6-HSA NP formulations after incubation in $10 \mathrm{mM}$ and $0.001 \mathrm{mM}$ glutathione for $18 \mathrm{~h}$. 


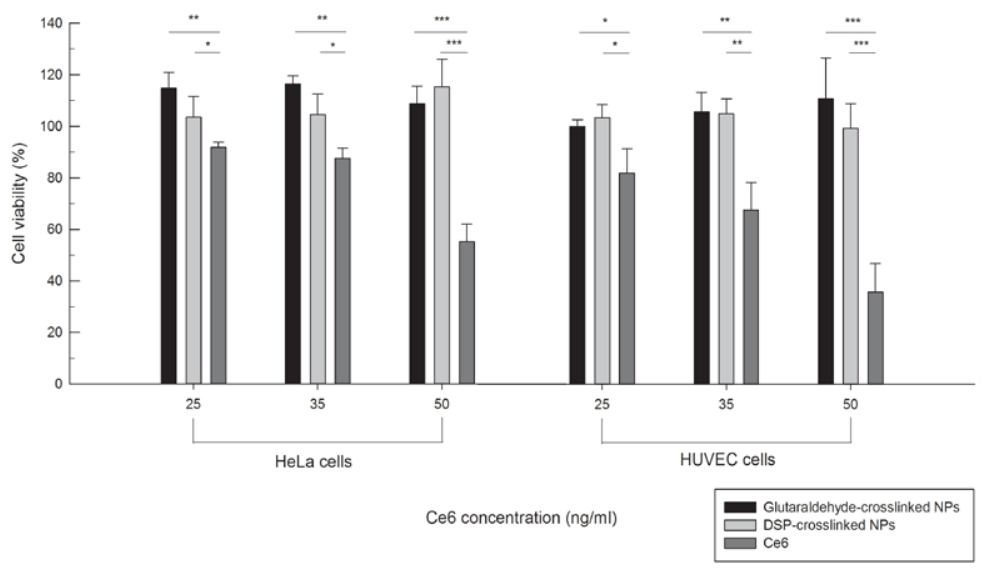

Figure 4: Dark toxicity in HeLa cells (A) and HUVEC cells (B) of the glutaraldehyde-cross-linked NP, the DSP-cross-linked NP, and the free $\mathrm{Ce} 6$ at different drug concentrations after a $24 \mathrm{~h}$ incubation. Asterisks indicate statistical significance with ${ }^{*} p<0.05$, ${ }^{* *} p<0.001$, and ${ }^{* * *} \mathrm{p}<0.0001$.

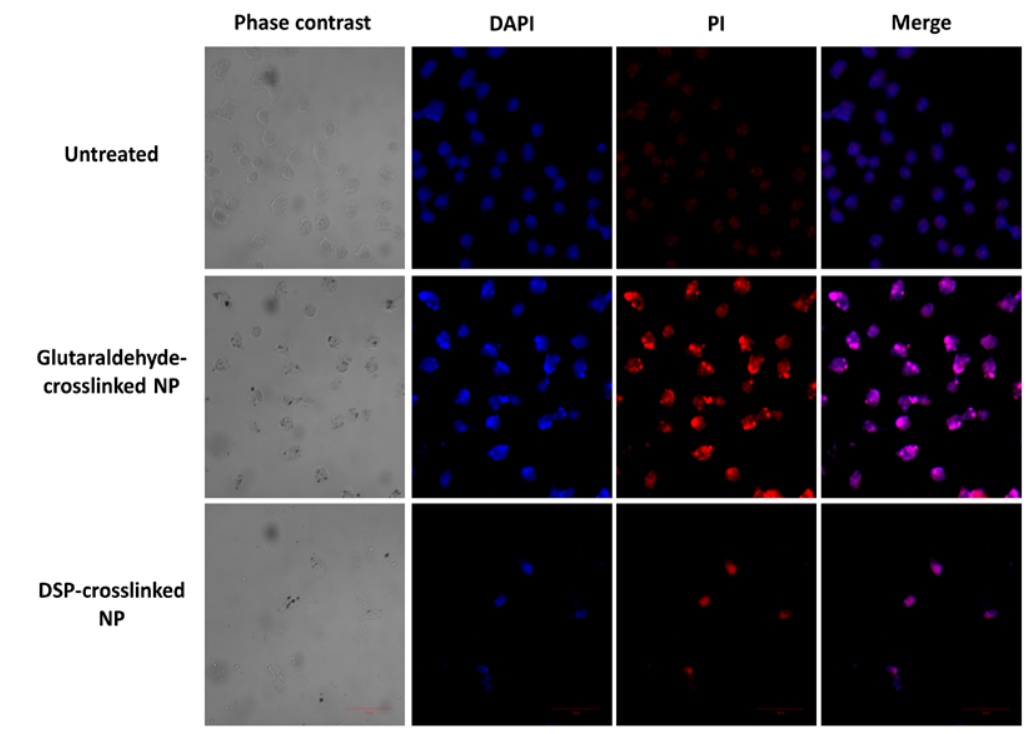

Figure 5: DAPI/PI-stained cells examined by confocal microscopy. The treated cells were incubated for $24 \mathrm{~h}$ with either the glutaraldehyde or the DSP-cross-linked Ce6-HSA NP and irradiated with a $660 \mathrm{~nm}$ LED lamp for $10 \mathrm{~min}$. The images show that both Ce6-NP cause cell death.

with both the glutaraldehyde and the DSP-cross-linked Ce6-HSA $\mathrm{NP}$, adjusted to a drug concentration of $5 \mathrm{ng} / \mathrm{ml}$. After 24 hours of incubation, the co-localization of DAPI and PI was determined. The PI internalization is representative of highly condensed and fragmented chromatin in apoptotic cells [39]. Our results confirm qualitatively that, after exposure to light, both drug-containing NP induce cell death (Figure 5). In contrast, untreated cells presented no indication of apoptosis, as can be observed by the lack of intense red fluorescence due to PI internalization. In order to quantitatively determine the cell viability difference between the glutaraldehyde and the DSP-crosslinked Ce6-HSA NP, dose-response curves were constructed after incubation for 1,6 , and $24 \mathrm{~h}$. All cells were irradiated to induce photo toxicity immediately after incubation. After 1 and $6 \mathrm{~h}$ of incubation, no significant difference was observed between the death inductions by either type of Ce6-HSA NP. However, after $24 \mathrm{~h}$ of incubation, a statistically significant difference between the Ce6-HSA NP with the different cross-linkers was apparent (Figure 6). The DSP-cross-linked Ce6-HSA NP was able to decrease the cell viability by at least $20 \%$ more than the glutaraldehyde-cross-linked NP in the $5-15 \mathrm{ng} / \mathrm{ml} \mathrm{Ce} 6$ concentration range. Even though both types of Ce6-HSA NP showed an increase in death induction with longer incubation times, possibly due to proteolytic degradation of the NP leading to drug activation, the DSP-cross-linked NP were much more efficiently activated by $24 \mathrm{~h}$ of incubation. This result indicates an improvement in the drug delivery function mediated by redox-sensitivity, particularly after longer doselight intervals and at lower concentrations.

Next, we wanted to qualitatively evaluate the internalization mechanism and localization of the NP within the HeLa cells. For this purpose, we labeled both types of NP with amine-reactive fluorescein isothiocyanate (FITC) and co-incubated them with FM4-64, an endosomal marker. The drug concentration was adjusted to $10 \mathrm{ng} /$ $\mathrm{ml}$ and the cells were not exposed to light to prevent photo toxicity. 

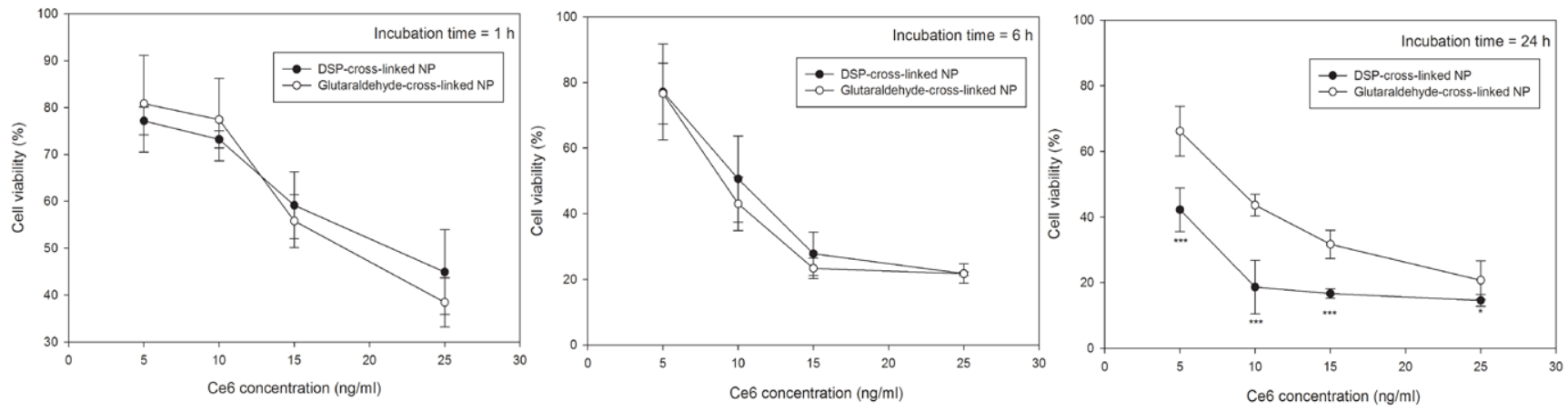

Figure 6: HeLa cell viability after incubation with glutaraldehyde or DSP-cross-linked NP as a function of the Ce6 concentration after 1,6 , and 24 h of incubation. Two-tailed t-test was used to establish significance at each individual time point; Asterisks indicate statistical significance with ${ }^{*} p<0.05$ and ${ }^{* * *} p<0.0001$.

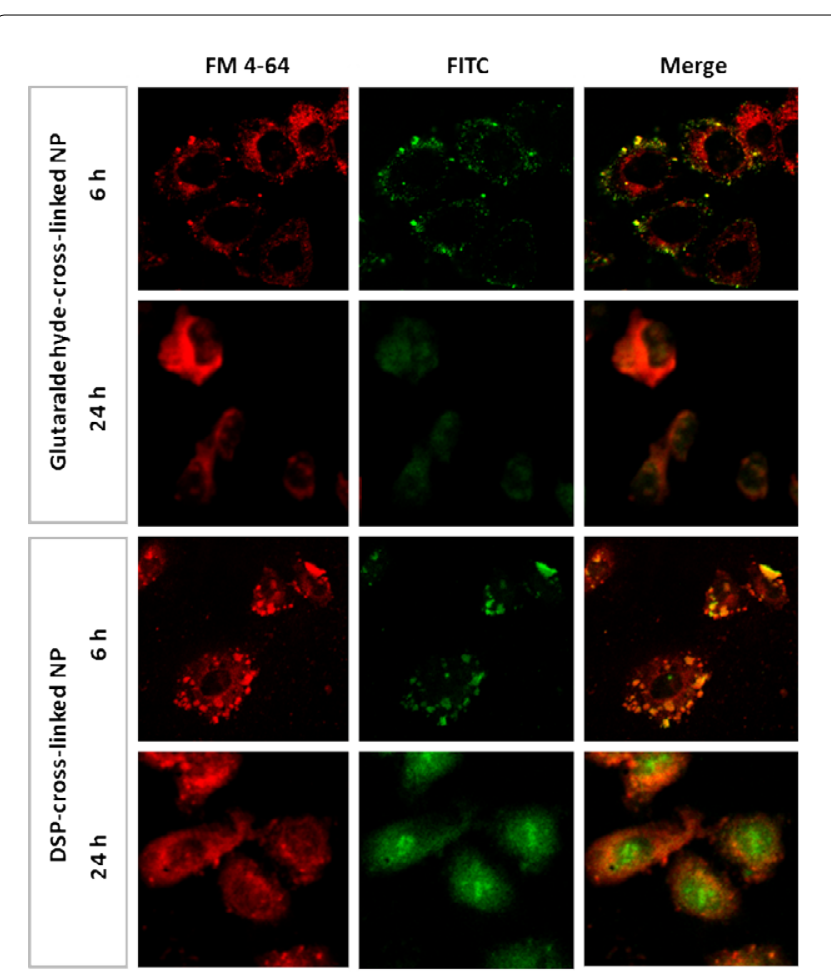

Figure 7: Qualitative examination of the endosomal escape of glutaraldehyde and DSP-cross-linked HSA-Ce6 NP in HeLa cells by confocal microscopy. The cells were incubated with the NP adjusted to a drug concentration of $10 \mathrm{ng} / \mathrm{ml}$ for $6 \mathrm{~h}$ and were not exposed to light. Images show the cell autofluorescence (A), cells incubated with FM-4-64 to label the endosomes (B), cells exposed to FITC-labeled NP (C), and the merged image (D).

After $6 \mathrm{~h}$ of incubation, both, the glutaraldehyde and the DSP-crosslinked Ce6-HSA NP, appear to be co-localized with endosomes (Figure 7). After $24 \mathrm{~h}$, however, the fluorescence was distributed evenly throughout the cell. Both the redox-sensitive and the redox-unsensitive NP appeared to be equally internalized and behaved similarly after 6 and $24 \mathrm{~h}$ of incubation.

\section{Conclusion}

We designed and synthesized a smart redox-responsive system for PDT by stabilizing albumin NP using a thiol-cleavable cross- linker. The obtained NP had a suitable size, shape, and polydispersity to theoretically allow for tumor targeting. The fluorescence of the immobilized model photosensitizer Ce6 was successfully quenched when the NP were intact, and the quenching was shown to be inversely proportional to the generation of singlet oxygen, the main photo toxic agent of PDT. The designed system successfully prevented dark toxicity in healthy or non-targeted tissues, as shown in vitro. Furthermore, singlet oxygen production was low when the NP was intact. NP disintegration produced by reducing conditions caused increased singlet oxygen production and resulted in increased cell death upon radiation. Redox-sensitive cross-linking of the Ce6-HSA NP did not alter interactions with the cells in terms of internalization routes and subcellular localization. Altogether, our results provided evidence that our designed redox-responsive system is significantly more efficient in inducing cell death after longer dose-light intervals than the nonredox-responsive NP. Finally, this work provides a working model of how to improve existing tumor-targeted NP-based PDT.

\section{Acknowledgement}

This work was partly supported by the NIH RISE Grant 2R25GM061151-13 and the Institute for Functional Nanomaterials (NSF Cooperative Agreement 1002410).

\section{References}

1. Housman G, Byler S, Heerboth S, Lapinska K, Longacre M, et al. (2014) Drug resistance in cancer: an overview. Cancers (Basel) 6: 1769-1792.

2. Hanahan D, Weinberg RA (2011) Hallmarks of cancer: the next generation Cell 144: 646-674.

3. Philchenkov AA, Shishko ED, Zavelevich MP, Kuiava LM, Miura K, et al (2014) Photodynamic responsiveness of human leukemia Jurkat/A4 cells with multidrug resistant phenotype. Exp Oncol 36: 241-245.

4. Allison RR, Mota HC, Bagnato VS, Sibata CH (2008) Bio-nanotechnology and photodynamic therapy--state of the art review. Photodiagnosis Photodyn Ther 5: 19-28.

5. Allison RR (2014) Photodynamic therapy: oncologic horizons. Future Oncol 10 123-124.

6. Huang Z, Xu H, Meyers AD, Musani Al, Wang L, et al. (2008) Photodynamic therapy for treatment of solid tumors--potential and technical challenges. Technol Cancer Res Treat 7: 309-320.

7. De Rosa MC, Crutchley RJ (2002) Photosensitized singlet oxygen and its application. Coord Chem Rev: 233-234.

8. Josefsen LB, Boyle RW (2008) Photodynamic therapy: novel third-generation photosensitizers one step closer? Br J Pharmacol 154: 1-3. 
Citation: Molina AM, Morales-Cruz M, Benítez M, Berríos K, Figueroa CM, et al. (2015) Redox-Sensitive Cross-Linking Enhances Albumin Nanoparticle Function as Delivery System for Photodynamic Cancer Therapy. J Nanomed Nanotechnol 6: 294. doi:10.4172/2157-7439.1000294

Page 8 of 8

9. Shirasu N, Nam SO, Kuroki M (2013) Tumor-targeted photodynamic therapy. Anticancer Res 33: 2823-2831.

10. Lamch L, Bazylińska U, Kulbacka J, Pietkiewicz J, Bieżuńska-Kusiak K, et al. (2014) Polymeric micelles for enhanced Photofrin II @ delivery, cytotoxicity and pro-apoptotic activity in human breast and ovarian cancer cells. Photodiagnosis Photodyn Ther 11: 570-585

11. Danhier F, Feron O, Préat $V$ (2010) To exploit the tumor microenvironment: Passive and active tumor targeting of nanocarriers for anti-cancer drug delivery. J Control Release 148: 135-146.

12. Greish K (2010) Enhanced permeability and retention (EPR) effect for anticancer nanomedicine drug targeting. Methods Mol Biol 624: 25-37.

13. Maeda $H$ (2012) Macromolecular therapeutics in cancer treatment: the EPR effect and beyond. J Control Release 164: 138-144.

14. Torchilin $\mathrm{V}$ (2011) Tumor delivery of macromolecular drugs based on the EPR effect. Adv Drug Deliv Rev 63: 131-135.

15. Schroeder A, Heller DA, Winslow MM, Dahlman JE, Pratt GW, et al. (2011) Treating metastatic cancer with nanotechnology. Nat Rev Cancer 12: 39-50.

16. Gallo JM, Hung CT, Perrier DG (1984) Analysis of albumin microsphere preparation. Int J Pharm 22: 63-74.

17. Lin W, Coombes AG, Davies MC, Davis SS, Illum L (1993) Preparation of sub$100 \mathrm{~nm}$ human serum albumin nanospheres using a $\mathrm{pH}$-coacervation method. J Drug Target 1: 237-243.

18. Müller BG, Leuenberger H, Kissel T (1996) Albumin nanospheres as carriers for passive drug targeting: an optimized manufacturing technique. Pharm Res 13: 32-37.

19. Langer K, Balthasar S, Vogel V, Dinauer N, von Briesen H, et al. (2003) Optimization of the preparation process for human serum albumin (HSA) nanoparticles. Int J Pharm 257: 169-180.

20. Li L, Hitchcock AP, Cornelius R, Brash JL, Scholl A, et al. (2008) X-ray microscopy studies of protein adsorption on a phase segregated polystyrene/ polymethylmethacrylate surface. 2. Effect of $\mathrm{pH}$ on site preference. $\mathrm{J}$ Phys Chem B 112: 2150-2158.

21. Kratz F (2008) Albumin as a drug carrier: design of prodrugs, drug conjugates and nanoparticles. J Control Release 132: 171-183.

22. Chen K, Preuß A, Hackbarth S, Wacker M, Langer K, et al. (2009) Nove photosensitizer-protein nanoparticles for photodynamic therapy: photophysical characterization and in vivo investigations. Photochem Photobiol B 96: 66-74.

23. Wacker M, Chen K, Preuss A, Possemeyer K, Roeder B, et al. (2010) Photosensitizer loaded HSA nanoparticles. I: Preparation and photophysical properties. Int J Pharm 393: 253-262.
24. Preuss A, Chen K, Hackbarth S, Wacker M, Langer K, et al. (2011) Photosensitizer loaded HSA nanoparticles II: in vitro investigations. Int J Pharm 404: 308-316.

25. Lee SJ, Koo H, Jeong $H$, Huh MS, Choi $Y$, et al. (2011) Comparative study of photosensitizer loaded and conjugated glycol chitosan nanoparticles for cancer therapy. J Control Release 152: 21-29.

26. Spikes JD (1990) Chlorins as photosensitizers in biology and medicine. J Photochem Photobiol B 6: 259-274.

27. Zeisser-Labouèbe M, Mattiuzzo M, Lange N, Gurny R, Delie F (2009) Quenching-induced deactivation of photosensitizer by nanoencapsulation to improve phototherapy of cancer. J Drug Targ 17: 619-626.

28. Habeeb AF (1966) Determination of free amino groups in proteins by trinitrobenzenesulfonic acid. Anal Biochem 14: 328-336.

29. Kraljic I, El-Mohsni S (1978) A new method for detection of singlet oxygen inaqueous solution, Photochem Photobiol 28: 577-581.

30. Bose B, Dube A (2008) Photodynamic efficacy of chlorin p6: a pH dependent study in aqueous and lipid environment. J Photochem Photobiol B 93: 32-35.

31. Meerovich I, Muthukrishnan N, Johnson GA, Erazo-Oliveras A, Pellois JP (2014) Photodamage of lipid bilayers by irradiation of a fluorescently labeled cell-penetrating peptide. Biochim Biophys Acta 1840: 507-515.

32. Kumar R, Roy I, Ohulchanskky TY, Vathy LA, Bergey EJ, et al. (2010) In vivo biodistribution and clearance studies using multimodal organically modified silica nanoparticles. ACS Nano 4: 699-708.

33. Sun Y, Chen ZL, Yang XX, Huang P, Zhou XP, et al. (2009) Magnetic chitosan nanoparticles as a drug delivery system for targeting photodynamic therapy. Nanotechnology 20: 135102.

34. Langer K, Anhorn MG, Steinhauser I, Dreis S, Celebi D, et al. (2008) Human serum albumin (HSA) nanoparticles: reproducibility of preparation process and kinetics of enzymatic degradation. Int J Pharm 347: 109-117.

35. Hwang C, Sinskey AJ, Lodish HF (1992) Oxidized redox state of glutathione in the endoplasmic reticulum. Science 257: 1496-1502.

36. Connelly JP, Botchway SW, Kunz L, Pattison D, Parker AW, et al. (2001) Timeresolved fluorescence imaging of photosensitiser distributions in mammalian cells using a picosecond laser line-scanning microscope. J Photochem Photobiol A Chem 142: 169-175.

37. Gomer CJ (2010) Photodynamic Therapy Methods in Molecular Biology. Humana Press: New York, USA.

38. Nowis D, Makowski M, Stokłosa T, Legat M, Issat T, et al. (2005) Direct tumor damage mechanisms of photodynamic therapy. Acta Biochim Pol 52: 339-352.

39. Morales-Cruz M, Figueroa CM, González-Robles T, Delgado Y, Molina A, et al (2014) Activation of caspase-dependent apoptosis by intracellular delivery of Cytochrome c-based nanoparticles. J Nanobiotechnology 12: 33. 